\title{
Extraction of Least-Dispersive Ultrasonic Guided Wave Mode in Rail Track Based on Floquet-Bloch Theory
}

\author{
Maodan Yuan $\left(\mathbb{D},{ }^{1}\right.$ Peter $W$. Tse $\mathbb{D}^{\mathbb{D}},{ }^{2}$ Weiming Xuan, ${ }^{1}$ and Wenjin $X u^{1}$ \\ ${ }^{1}$ School of Electro-Mechanical Engineering, Guangdong University of Technology, Guangzhou 510006, China \\ ${ }^{2}$ Department of Systems Engineering and Engineering Management, City University of Hong Kong, Hong Kong, China
}

Correspondence should be addressed to Maodan Yuan; mdyuan@gdut.edu.cn

Received 7 November 2020; Revised 4 January 2021; Accepted 29 January 2021; Published 9 February 2021

Academic Editor: Pedro A. Costa

Copyright (C) 2021 Maodan Yuan et al. This is an open access article distributed under the Creative Commons Attribution License, which permits unrestricted use, distribution, and reproduction in any medium, provided the original work is properly cited.

Ultrasonic guided wave (UGW) has shown great potential in the field of structural health monitoring of rail tracks due to its longrange capability and full cross section coverage. However, the practical application of UGW has been hindered by the complicated signal interpretation because of the natures of multiple modes and dispersion. Therefore, it is desirable that the effective UGW modes with high excitability and least dispersion can be identified and extracted for practical applications. In this paper, a numerical study on the guided wave propagation was carried out on a standard rail with 56E1 profile. Firstly, Floquet-Bloch theory was applied to obtain the dispersion curves of guided wave in a rail. Then, a 3D FE model was built to investigate the UGW propagation along the rail within the frequency range of $0-120 \mathrm{kHz}$. Wavenumber-frequency analysis method was applied to decompose and identify the propagating UGW modes. With a carefully designed 2D bandpass filter, a specific mode W0 was extracted in the wavenumber-frequency domain. Finally, a frequency band sweep technique was also proposed to get the optimal frequency band to achieve a pure and least-dispersive UGW mode along the rail web. The proposed method provides an effective way to extract efficient UGW modes to assess the integrity of the rail track, as well as other waveguides with complex geometry.

\section{Introduction}

Ultrasonic guided wave (UGW) has continuously attracted researchers' attention in the fields of nondestructive testing (NDT) and structural health monitoring (SHM) in railway engineering [1-3]. Rose et al. firstly discussed the potential application of defect detection in rail based on UGW technology $[1,2]$. Since then, various researches have been conducted from theoretical study to engineering application. Yue et al. studied the dispersion characteristics and propagation properties of the guided wave along the rail foot [4]. Zhou et al. developed a 3D diagnostic imaging technique based on time-of-flight signal features [5]. The advantages of UGW include long-range capability, full coverage across the whole cross section of the rail track, and rapid inspection with few transducers. However, due to the complex cross section of rail track compared to the plate or pipe, there are much more UGW modes propagating along the rail [6]. Moreover, most UGW modes are dispersive, making the interpretation of ultrasonic signals very difficult in practical applications. Therefore, of the first but not the least importance, the knowledge of dispersion curves and corresponding mode shapes should be obtained before applying UGW for rail inspection system [7, 8]. The dispersion curves contain the information regarding how available wave modes propagate. This information can help to explain the results and provide guidance for sensor deployment $[9,10]$.

So far, there are various methods developed by researchers to calculate the dispersion curves of UGW. Generally, available methods can be categorized as analytical, semianalytical, and numerical methods. Among them, analytical method has been developed only for the waveguides with simple cross section, such as plates and pipes. But when the geometry of waveguides becomes as complex as a rail track, it is impossible to obtain the dispersion curves analytically. Thus, numerical techniques have been developed. Nowadays, one of the most popular techniques to calculate the dispersion curves for waveguides with complex 
cross section is semianalytical finite element (SAFE). Instead of handling the three-dimension problem directly, it only requires two-dimension discretization for the cross section plane $[7,11,12]$. The SAFE method can efficiently solve the eigenvalue problem in terms of wavenumber at a given frequency, or in terms of frequency at a given wavenumber. Recently, efforts were also made to implement SAFE method in the commercially available finite element package [13]. Ramatlo et al. built a hybrid method coupling a 3D FEM model of the arbitrary discontinuity with two SAFE models of the rail to represent the semi-infinite incoming and outgoing waveguides [14]. Still, the main disadvantage is that it is difficult to include periodically discrete supports due to the analytical description of the wave propagation along the rail.

An alternative approach to calculate dispersion curves is wave finite element (WFE) method. It has been developed to handle the waveguide with appropriate boundary conditions based on commercial finite element (FE) packages. Thompson developed FE methods with a short length of rail with periodic condition to investigate the wheel-rail noise generation, but this method failed at the range of high frequencies [15]. Later, Ryue et al. investigated wave propagating in a rail track up to $80 \mathrm{kHz}$ with symmetric and antisymmetric boundary conditions $[16,17]$. Sanderson and Smith proposed a three-dimension FE model based on eigenfrequency analysis to obtain the dispersion curves of rail [18]. In these methods, however, the process should be repeated by changing the length of unit cell until all the modes are obtained. The process of WFE to calculate dispersion curves can be improved and accelerated based on the Floquet-Bloch (FB) theory. It controls periodic coefficients to trace the eigenfrequencies with varying wavenumber; thus, there is no need to change the length of rail section [19]. The FB theory has been applied for dispersion analysis of different periodic systems, especially for the photonic crystal structures [20, 21]. There are two remarkable merits by applying FB theory. Firstly, the full advantages of existing commercial FE packages can be maintained, including available element types, material libraries, and well-developed iterative numerical algorithms. Thus, there is no need to define element and formulate FE process. Secondly, even complex rail system with supporting layers, such pads and sleepers, can generally be modelled without additional difficulties. To the best of the authors' knowledge, there is still no prior study on applying FB theory to effectively calculate dispersion curves for rail tracks.

Furthermore, to develop UGW inspection or monitoring system, it is desirable to excite a single UGW mode in a well-controlled direction. A narrowband signal should be excited after dedicated frequency tuning and tailor-made sensor deployment [22]. Loveday proposed phased array design of four ultrasonic transducers to control over which modes were excited and sensed preferentially in one direction along the rail $[3,23]$. Their system requires permanent sensors attachment with accurate phase delay, but extra propagating modes still exist. Xing et al. presented a mathematical model composed of a modal vibration factor and a modal orthogonal factor to select a suitable UGW mode for detecting a specific crack on a rail [24]. However, arrayed transducer deployment and excitation is not always available for the uneven and inaccessible surface of rails. Moreover, the closeness of dispersion curves and mode repulsion makes it practically difficult to exclude unwanted modes [25]. Even more UGW modes may show up due to mode conversion at discontinuity. Therefore, complex UGW signals with multiple modes are much more common. To apply such UGW signal for defect detection in rail track, the first priority is to identify and extract the effective propagating modes. Loveday et al. proposed a mode extraction method based on the orthogonality of mode shapes in rail with laser vibrometer [6]. This method was verified for the excitation with a continuous sinusoidal wave at a single frequency. However, a short pulse excitation is more common and single wave mode information should be extracted from such wide bandwidth. Alleyne proposed two-dimension Fourier transform (2D-FFT) to analyse propagating multimodal Lamb waves in the wavenumberfrequency domain and identified individual Lamb wave modes [26]. By applying a proper 2D bandpass filter in the wavenumber-frequency domain, Tian and $\mathrm{Lu}$ decomposed wave modes in the plate and extracted single-mode Lamb wave [27]. Inspired by the filtering method in plates, the broadband UGW signal in the rail excited by conventional ultrasonic transducer or laser pulse can be analysed in the wavenumber-frequency domain. However, there are much more UGW modes existing in rail, compared to Lamb wave in plates. It leads to the fact that dispersion curve of adjacent mode is very close. Therefore, careful study on the mode identification and extraction should be carried out in rail, which has not been discussed yet.

In this paper, we will focus on the study of extraction of efficient ultrasonic guided wave mode in rail track based on Floquet-Bloch theory. The paper will be organized as follows. The numerical implementation and the obtained dispersion curves based on FB theory are presented in Section 2. Basic theory for extracting specific UGW modes from broadband excitation is introduced in Section 3. Numerical experiments based on 3D FE model are carried out to illustrate the process to extract a least-dispersive UGW mode along rail web, as described in Section 4. Finally, conclusions are drawn in Section 5.

\section{Dispersion Curves Calculation Based on Floquet-Bloch Theory}

2.1. Numerical Implementation of FB Theory. To facilitate the dispersive analysis in the rail, FB theory is applied in this study and implemented in a commercial FE software in COMSOL Multiphysics. A unit cell of rail with a small segment is chosen for calculation, whose length is determined by the maximum frequency of interest. There is a maximum value for the incident wavenumber, corresponding to a minimum wavelength $\lambda_{\min }=2^{*} L$, where $L$ is the length of unit cell. FB periodic boundary conditions are 
applied to both ends along the direction of wave propagation to make this model an infinite structure, and FB is defined as

$$
u_{d s t}=u_{s r c} e^{-i k_{i} \cdot\left(r_{d s t}-r_{s r c}\right)},
$$

where $u$ is the displacement and $r$ is the distance; dst and src refer to the destination and source plane, respectively; and $k_{i}$ refers to incident wavenumber. With an eigenfrequency study in a structural module, the natural frequencies and mode shapes are calculated for a given incident wavenumber $k_{i}$. By sweeping wavenumber $k_{i}$, all the eigenfrequencies are obtained. The resulting wavenumber-frequency pairs form $k-f$ dispersion relation, which can be further converted to dispersion curves regarding phase velocity $c p-f$ and group velocity $c g$ - $f$. The general process to calculate dispersion curves based on FB theory is illustrated in Figure 1.

$$
\begin{aligned}
& c_{p}=\frac{\omega}{k}, \\
& c_{g}=\frac{\partial \omega}{\partial k} .
\end{aligned}
$$

2.2. Dispersion Curves of Guided Wave in a Rail Track. A standard rail with the profile of 56E1 was chosen for the study here, and a three-dimension model was designed and built. Figure 2 shows the cross section of rail 56E1 and threedimension geometry of the designed model. In this work, the discrete supports, such as rail pad and sleeper, will not be considered to avoid the uncertainty in determining wavenumber-frequency pair at high frequency [16]. FB boundaries were set at both ends of the rail and other boundaries were stress-free. Moreover, there are two approaches to reduce the computation cost for rail by applying higherorder elements and geometrical symmetry if applicable [28]. In the convergence study, mesh size of commonly used quadratic element should be chosen as 10 nodes per wavelength, while approximately half the wavelength is enough for quartic (4th order) element. Therefore, quartic Lagrange elements available in COMSOL Multiphysics were chosen to minimize the element number and save computation amount. Apart from this, the introduction of symmetric and antisymmetric boundary was applied to categorize all the UGW modes into two separate groups. In this case, the simple linear elastic material model was taken into consideration. The material properties are listed in Table 1.

Followed by the process as Figure 1, the dispersion curve can be obtained for this rail. The length of unit cell was set as $L=10 \mathrm{~mm}$ to calculate the exact UGW modes in the frequency up to $120 \mathrm{kHz}$. Here, only the first 60 wave modes were calculated at the frequency range of $0-120 \mathrm{kHz}$, which covers the frequency of interest in UGW for the rail. The corresponding dispersion curves regarding phase velocity and group velocity are as shown in Figure 3. There are thousands of points occupying the $f-k$ domain, corresponding to various wave modes consisting of the dispersion curves. At the upper right in Figure 3(a) and bottom right in Figure 3(b), the missing curves imply that there are more than 60 modes at frequencies higher than $80 \mathrm{kHz}$. The results indicate that almost numerous kinds of UGW modes could possibly exist in a rail under different boundary conditions and proper excitations. Moreover, because there are much more UGW modes than those of well-known waveguides, such as plate and pipes, the UGW modes are very close to each other. Such closeness of adjacent modes makes it difficult to identify and separate specific UGW mode in rail.

From the observation of the dispersion curves, several conclusions can be drawn. Firstly, when the frequency is increasing, all the wave modes become less dispersive and converge to a relatively low velocity, which is very close to surface wave speed. Therefore, high-order UGW mode cluster in high frequency has high potential for small defect detection in a rail. Secondly, high-order UGW modes are more dispersive than those of the low-order ones at a certain frequency, implying that low-order modes are much more preferential for UGW long-range application in rail tracks.

\section{Basic Theory for Extraction of Specific UGW Mode}

3.1. Frequency Wavenumber Decomposition. In this section, general procedures to extract a specific UGW mode from broadband excitation will be illustrated. At first, frequency wavenumber decomposition will be introduced to identify a specific mode. Then, frequency band sweep is illustrated to choose the optimal frequency from broadband. To transform the time-space wavefield $u(t, x)$ into the wavenumber-frequency domain, two-dimension fast Fourier transform is required. The corresponding wavefield $U(f, k)$ in wavenumber-frequency domain can be written as [26]

$$
U(f, k)=\sum_{x=x_{1}}^{x=x_{m}} \sum_{t=t_{1}}^{t=t_{n}} u(t, x) e^{-j(2 \pi f t-k x)} \Delta t \Delta x,
$$

where $x=x_{1}, \ldots, x_{m}$ is the space vector; $t=t_{1}, \ldots, t_{n}$ is the time step; $f$ is the frequency; $k$ refers to the wavenumber vector; and $\Delta x$ and $\Delta t$ are the spatial and temporal intervals, respectively. The wavefield in wavenumber-frequency domain can effectively identify and separate the different UGW modes. Then, window functions can be applied to filter out the unwanted components.

Thus, if a $2 \mathrm{D}$ bandpass filter $W(f, k)$ is designed, the filtered spectrum in wavenumber-frequency domain is [27]

$$
U_{\text {filter }}(f, k)=U(f, k) W(f, k),
$$

where $U(f, k)$ refers to the full spectrum. 2D filter $W(f, k)$ is designed around the dispersion curve of the desired UGW mode to retain the desired components of the spectrum. Therefore, the center of the $2 \mathrm{D}$ filter is set to be the theoretical dispersion curve of the desired UGW mode. Then, the major remaining energy is the desired UGW mode. Specifically, the $2 \mathrm{D}$ bandpass filter can be designed as

$$
W(f, k)=W_{f}(f) W_{k}(f, k),
$$

where $W_{f}(f)$ is a frequency band, corresponding to the excitation by sensors, and $W_{k}(f, k)$ is a wavenumber band 


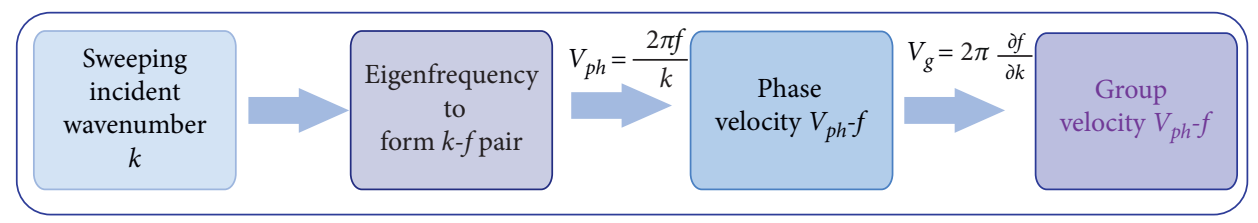

Figure 1: The process to calculate dispersion curves based on FB theory.

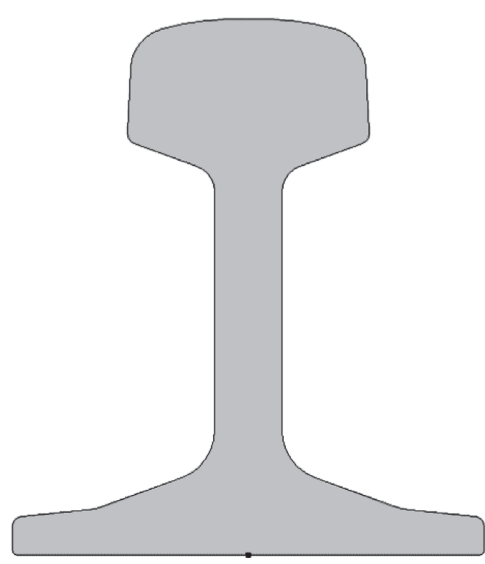

(a)

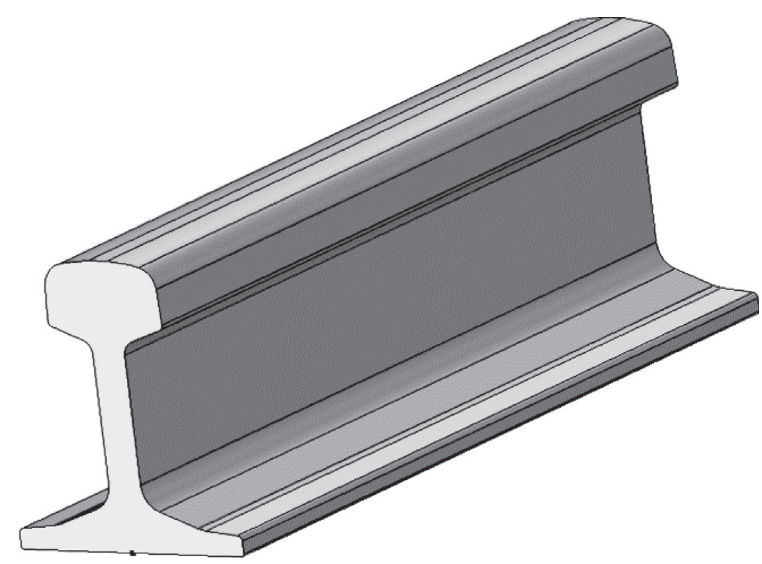

(b)

FIgURE 2: Geometry model of a rail track. (a) Cross section and (b) 3D geometry.

TABLE 1: Material property of the rail.

\begin{tabular}{lcc}
\hline Young's modulus, $E(\mathrm{GPa})$ & Poisson's ratio, $v$ & $\begin{array}{c}\text { Mass density, } \rho \\
\left(\mathrm{kg} / \mathrm{m}^{3}\right)\end{array}$ \\
\hline 207 & 0.33 & 7850 \\
\hline
\end{tabular}

centered at the theoretical dispersion curve $k(f)$ of the desired UGW mode. After the filtering process, the filtered spectrum can be transformed back by an inverse Fourier transform (IFFT) to reconstructed space-time domain signal $u_{\text {filter }}(t, \mathrm{x})[27]$ :

$$
u_{\text {filter }}(t, x)=\frac{1}{2 \pi} \sum_{k=k_{1}}^{k=k_{m}} \sum_{f=f_{1}}^{f=f_{n}} U_{\text {filter }}(f, k) e^{j(2 \pi f t-k x)} \Delta f \Delta k
$$

where $\Delta f$ and $\Delta k$ are the frequency and wavenumber intervals, respectively. Therefore, individual modes can be decomposed and reconstructed from the general UGW signals based on wavenumber-frequency analysis.

3.2. Frequency Band Sweep. For a UGW system with broadband excitation, each mode is generally dispersive. To obtain a less dispersive UGW signal, a specific frequency band should be determined for receiving sensing system. Then, this frequency band can be adopted as $W_{f}(f)$ in the 2D $f$ - $k$ filter $W(f, k)$. Thus, effective UGW propagating along the rail is acquired. In the case of arbitrary excitation, the most favorable frequency band is not known a priori. Therefore, a sweep study on the frequency band $\left(f_{l}, f_{u}\right)$ in the range of interest should be implemented for practical applications.
The frequency band $\left(f_{l}, f_{u}\right)$ can be obtained by using the simple bandpass filter:

$$
\begin{aligned}
W_{f}(f) & = \begin{cases}1, & f_{l} \leq f \leq f, \\
0, & \text { else, }\end{cases} \\
f_{l} & =f_{\text {min }}, f_{u}=f_{\text {min }}+N_{f} \Delta f,
\end{aligned}
$$

where $f_{l}$ and $f_{u}$ are the lower and upper frequency limits, $\Delta f$ is frequency resolution, and $N^{*} \Delta f$ is the frequency bandwidth of the receiving system. The frequency band sweep is carried out by sweeping lower frequency limit and frequency bandwidth. For each frequency band $\left(f_{l}, f_{u}\right)$, the relative amplitude is calculated using equation (4) for a distant position. The maximum amplitude indicates the most sensitive frequency band to sense the UGW mode. With a procedure of the frequency band sweep, the frequency range of the receiving systems can be determined for the extraction of efficient UGW. This methodology can be applied to determine the sensor bandwidth and the filter in laser-based UGW system.

\section{3D FE Modelling of Wave Propagation in Rail Track}

4.1. 3D FE Model Setup. Transient guided waves propagation in rail is of importance to provide a visual and comprehensive way to study the case-based UGW modes in rails. Different UGW modes can be studied by a certain combination of boundary condition and excitation. In this study, a three-dimension FE model was established to simulate broadband UGW by commercial finite element software Abaqus, shown in Figure 4. The length of the investigated 


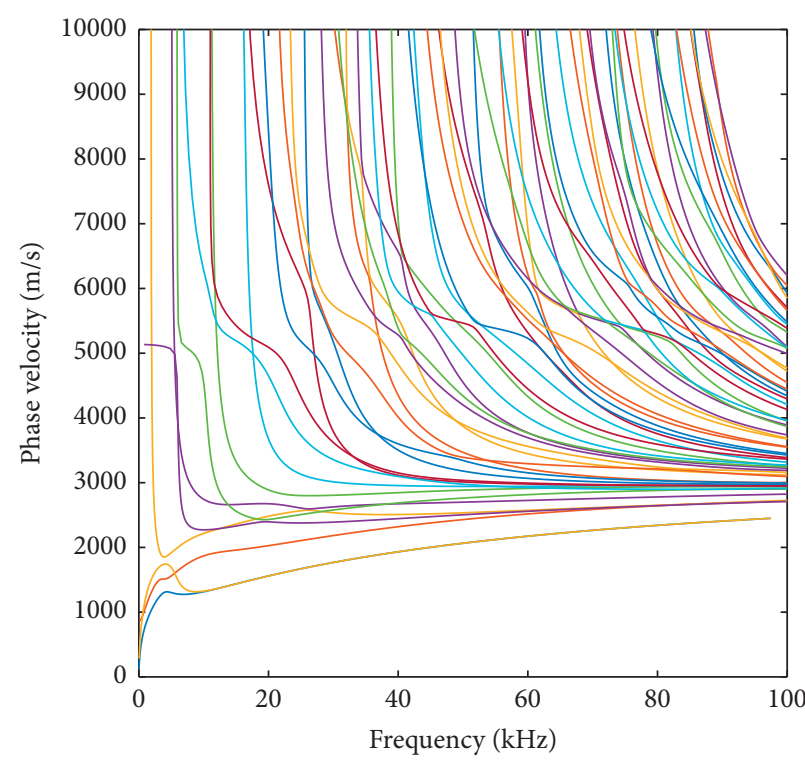

(a)

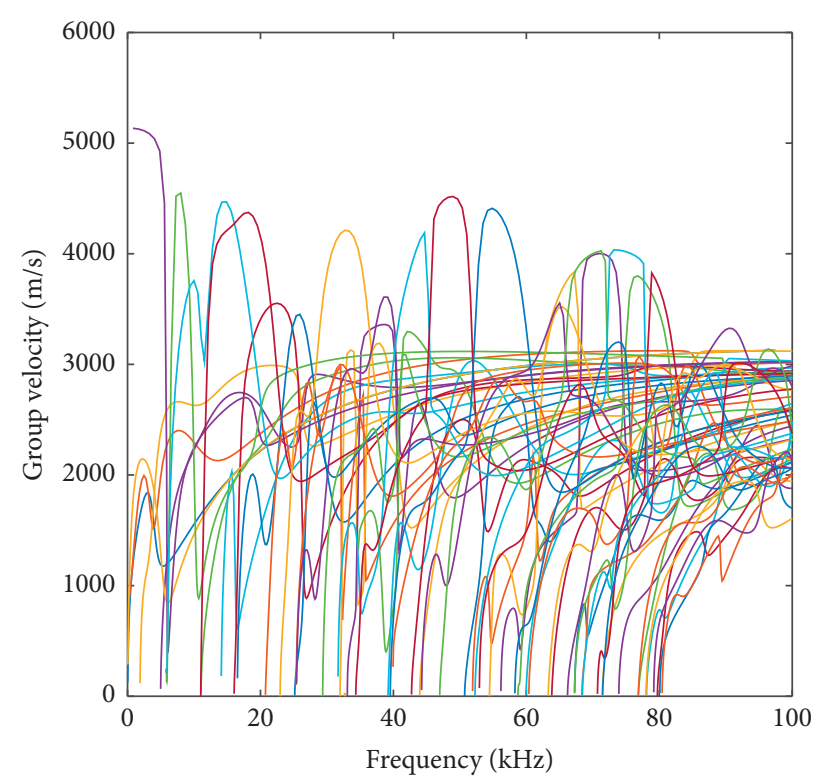

(b)

FIGURE 3: Dispersion curves for rail 56E1: (a) phase velocity and (b) group velocity.

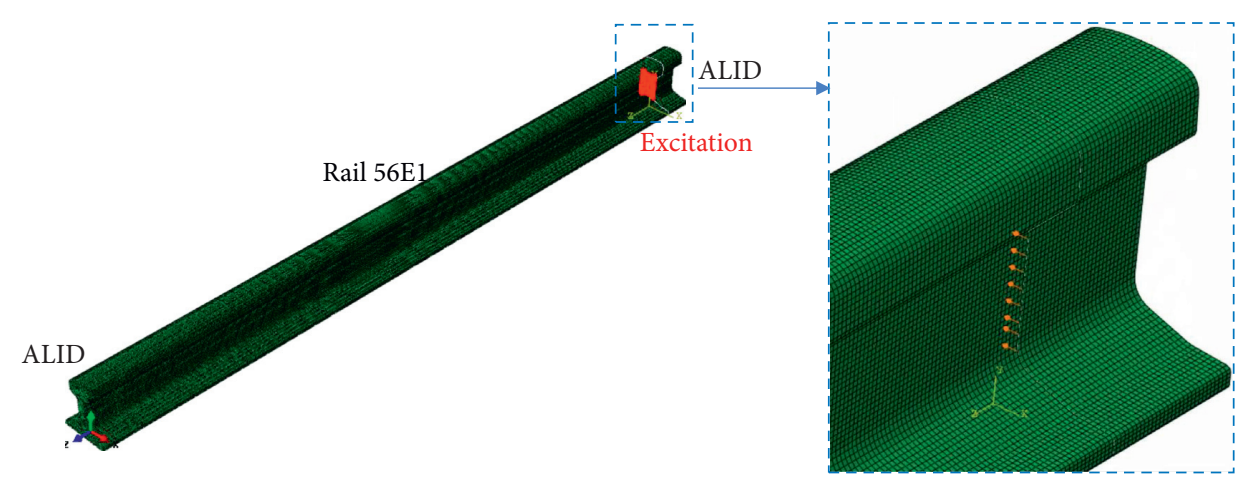

Figure 4: FE model for wave propagation in a rail.

rail is $2 \mathrm{~m}$. A central difference method to integrate the equations of wave motion explicitly through time is adopted in the Abaqus/Explicit solver. The kinematic conditions at one increment can be calculated by previous increment. This explicit method is especially well-suited to solve high-speed dynamic events such as elastic wave propagation. In consideration of generating ultrasonic waves in rail with a broadband up to the frequency of $120 \mathrm{kHz}$, a short pulse with almost flat response within the frequency range was used as the excitation signal, which is shown in Figure 5. Compared to point source generation, line-source generation was better at creating a directed surface wave to give a strong indication to the presence of a surface flaw [29]. Therefore, in the study, a pulse excitation was applied on a line at the rail web with displacement boundary condition.

Element size is essential to achieve adequate accuracy in FE based wave propagation modelling. Courant-Friedrichs-Lewy (CFL) condition should be satisfied that element sizes are small enough to calculate the wave propagation of the highest frequency. To ensure enough accuracy of calculation, we chose the element size as 10 nodes per minimum wavelength, i.e., surface wavelength at the largest frequency. Consider that tens of kilo Hertz is the typical frequency range in UGW testing in rails; this work focused on the frequency range from $20 \mathrm{kHz}$ to $120 \mathrm{kHz}$. On this basis, element size of $3 \mathrm{~mm}$ was adopted. To reduce the size of the whole model and to minimize the multiple end reflections and mode conversion, absorbing layers with increasing damping (ALID) were applied at both ends of the rail [30]. There were 30 successive layers added for both ends, symmetrically. The thickness of each layer was $3 \mathrm{~mm}$, which was equal to the element size. Stiffness proportional damping was set as 0 to limit the effects on stable time increment. Mass proportional damping across absorbing layers was set as $\alpha(x)=\alpha_{\max } x^{p}$. The maximum damping was set as $\alpha_{\max }=1 \times 10^{7}$ and $p$ was set to be 3 . The whole model was meshed with C3D8R element. Consequently, there are 692831 elements and 774530 nodes. The total number of variables in the model is 2323590 . The time increment was chosen as the 


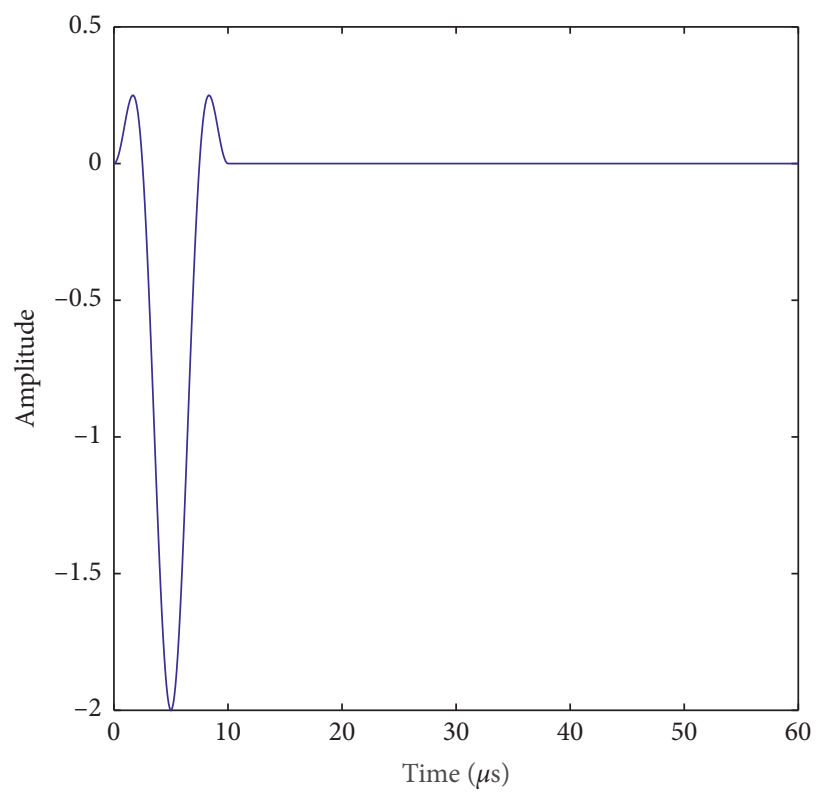

(a)

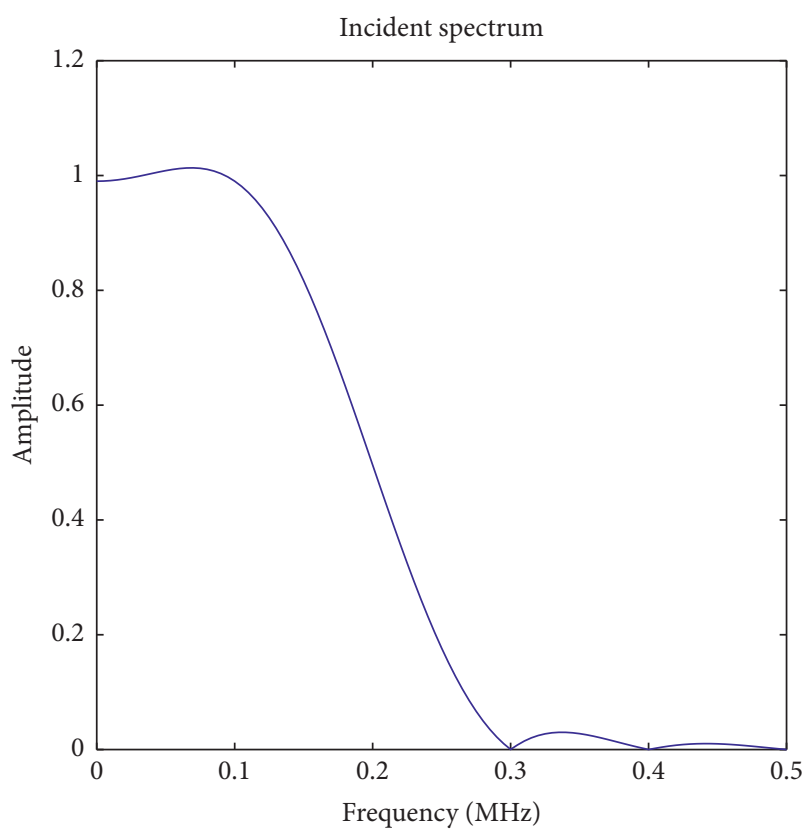

(b)

FIgURE 5: Broadband excitation signal: (a) A-scan waveform and (b) its spectrum.

default value, because the software automatically calculated the stable time step according to the minimum mesh size.

4.2. UGW Propagation in Rail. The UGW propagation along rail at different moments, $t=0.01 \mathrm{~ms}, 0.4 \mathrm{~ms}$, and $0.8 \mathrm{~ms}$, is shown in Figure 6. From the results, there are serval obvious wave modes propagating along rail head, rail web, and rail foot, simultaneously. The main energy is focused along the rail web. To identify the existing UGW modes, linear receivers were applied along the center of rail web. Figure 7(a) shows B-scan signal of the wavefield along the rail web. The contour band represents the out-of-plane displacement in $x$ direction, which could be easily measured by laser vibrometers in practical applications. It is clear to see that the waveform is constantly changing with the propagation distance as expected due to the multimodal and dispersive nature. It is difficult to identify each UGW mode and separate them in time-space wave field. Therefore, 2D Fourier transform was applied to convert the time-space wavefield into wavenumber-frequency domain, shown in Figure 7(b). The horizontal axis is the positive frequency and vertical axis is the positive wavenumber. From the results in wavenumber-frequency domain, UGW modes can be discernible. In order to identify the existing UGW modes in rails, the theoretically predicted dispersion curves in Section 2 were applied for comparison. The results show that the $f-k$ spectrum matches well with the predicted dispersion curves based on FB theory. Although there are numerous UGW modes observed within $0-120 \mathrm{kHz}$, only several dominant wave modes can be clearly identified. Specifically, the most dominant mode with most excited energy, here denoted as W0, can be clearly observed and separated from other modes. Because this W0 mode possesses most of the energy, it is reasonably considered to be the effective propagating UGW mode along the rail web. Therefore, W0 mode should be well extracted for the application of SHM for rails.

4.3. Extraction of Least-Dispersive UGW Mode. A $2 \mathrm{D} f-k$ bandpass filter is designed as equation (5), while the frequency filter $W_{f}(f)=1$ is across all the frequency and the wavenumber filter $W_{k}(f, k)$ is designed as $W_{k}(f, k)=0.5\left[1+\cos \left(|k-k(f)| / D_{w k}\right)\right]$. Considering the closeness of various UGW modes in rails, high resolution in $f-k$ domain is required to separate all the modes. Therefore, the wavenumber bandwidth $D_{w k}$ should be chosen as small as possible to exclude other modes. Here, a small value is chosen as $D_{w k}=20 \mathrm{~m}$. Moreover, the wavenumber increment is reduced to $0.5 \mathrm{~m}$ via polynomial interpolation to match with the $\mathrm{k}-\mathrm{f}$ result from FE simulation. Therefore, the dominant mode W0 was filtered and the resulting spectrum for retaining the dominant mode is shown in Figure 8(a). This dominant UGW mode along rail web can also be confirmed from the dispersion analysis of rail track in Section 2 and the 3D mode shape of $\mathrm{W} 0$ at $60 \mathrm{kHz}$ is shown in Figure 7. It is clear to see that most energy propagates along the rail web and the peak displacement appears at the central line.

Although a pure mode W0 is successfully extracted from the original multimodal signal, the dispersion curve is nonlinear in the frequency of $0-120 \mathrm{kHz}$, especially at low frequency around $20 \mathrm{kHz}$. This nonlinearity at low frequency will make the UGW waveform change along the propagation distance. Therefore, in order to effectively apply mode W0 for SHM application in rails, a specific frequency band of the excitation and receiving system should be defined to extract the nondispersive or least-dispersive part. To choose the 


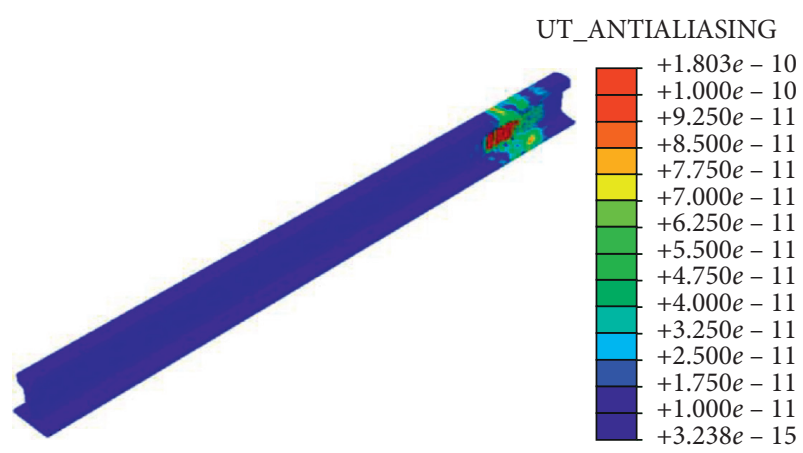

(a)

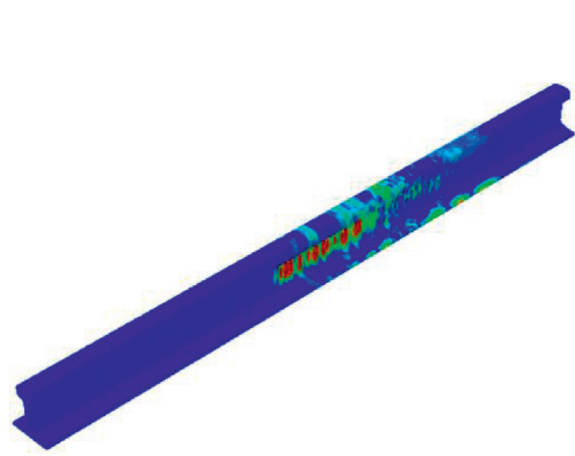

(b)

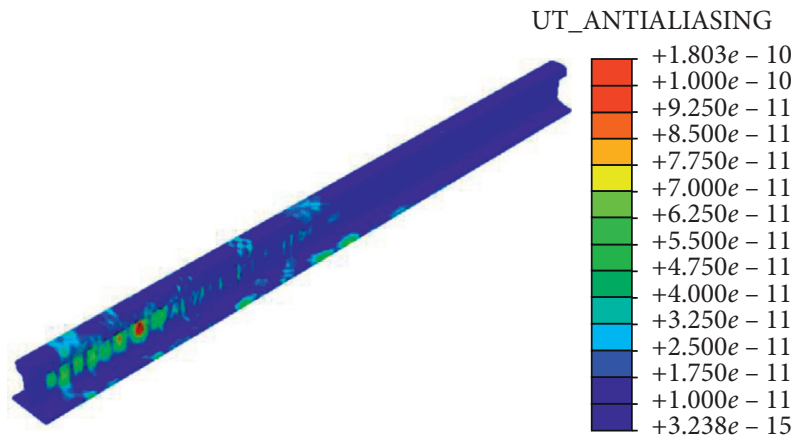

(c)

FIgURE 6: Wave propagation in a rail at time of (a) $t=0.01 \mathrm{~ms}$, (b) $t=0.4 \mathrm{~ms}$, and (c) $t=0.8 \mathrm{~ms}$.

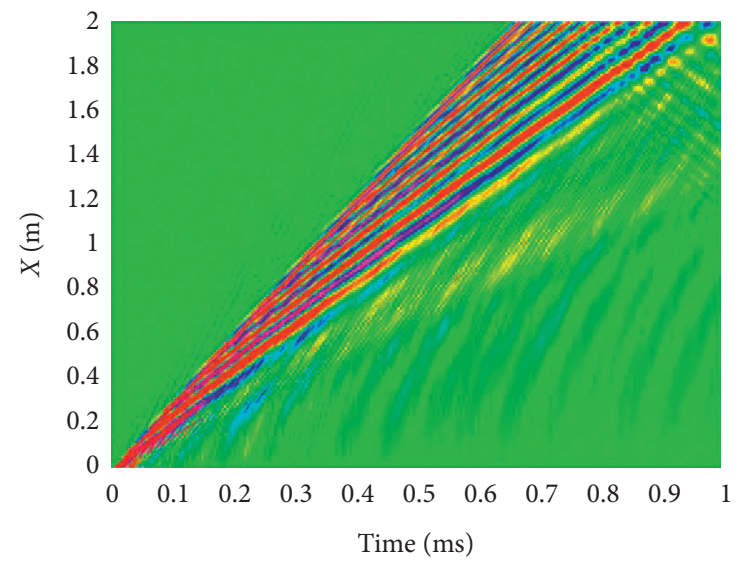

(a)

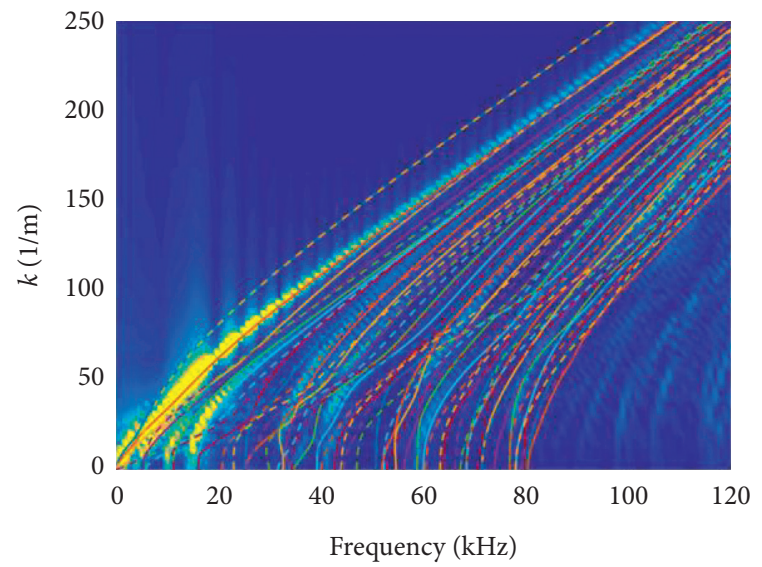

(b)

FIGURE 7: UGW signal from the rail web in (a) time-space domain and (b) wavenumber-frequency domain compared with theoretical dispersion curves.

specific frequency band for sensing system, a frequency band sweep was applied to investigate the effective amplitude of the reconstructed signal. The original signal was received at $1 \mathrm{~m}$ away from the excitation position. The initial frequency was set as $20 \mathrm{kHz}$, because our main focus is the field of ultrasonics. The amplitudes were normalized with the maximum value, and the result is as shown in Figure 9. The higher the amplitude, the more effective the UGW propagate along the rail web. From the results, the effective amplitude was obtained with a frequency band of $25-120 \mathrm{kHz}$, corresponding to the least-dispersive part of dominant mode W0.

Therefore, with the selected frequency band of $25-120 \mathrm{kHz}$, the spectrum was filtered as in Figure 10(a). To observe the wavefield of the single UGW mode W0 in spacetime domain, the filtered spectrum of W0 was then transformed using the inverse 2D FFT. Figure 10(b) gives the comparison between the original full wavefield and the 


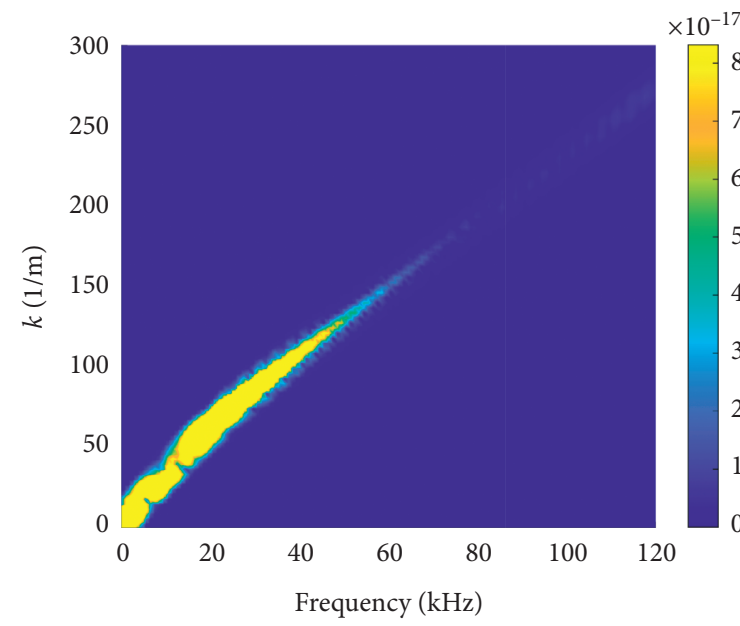

(a)

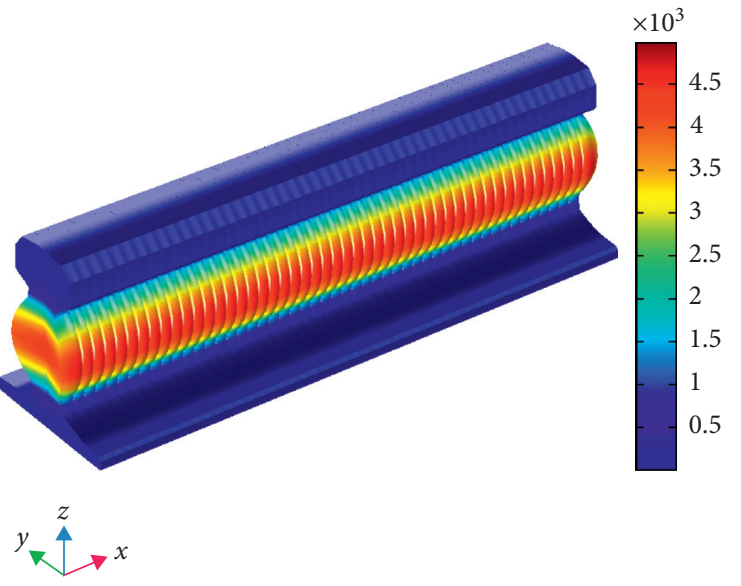

(b)

Figure 8: UGW mode W0 identification: (a) filtered spectrum and (b) 3D mode shape of W0 at $60 \mathrm{kHz}$.

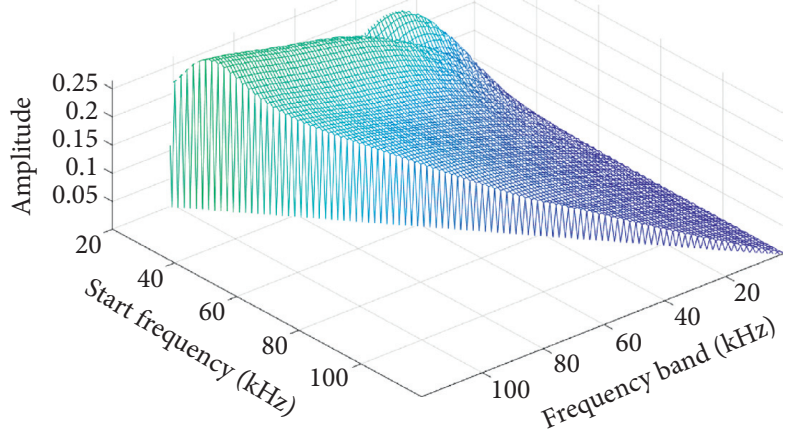

FIGURE 9: Reconstructed amplitude with sweeping frequency band.

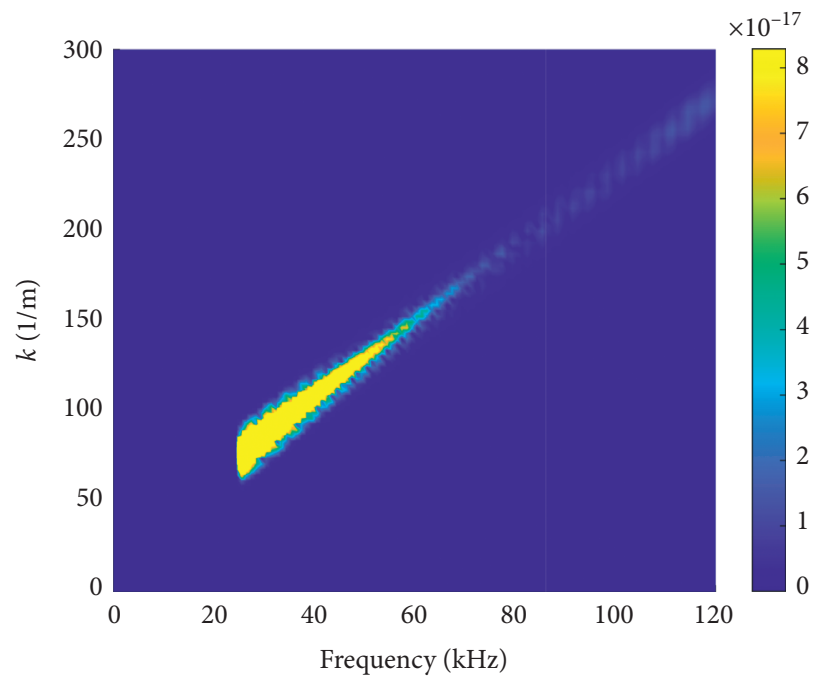

(a)

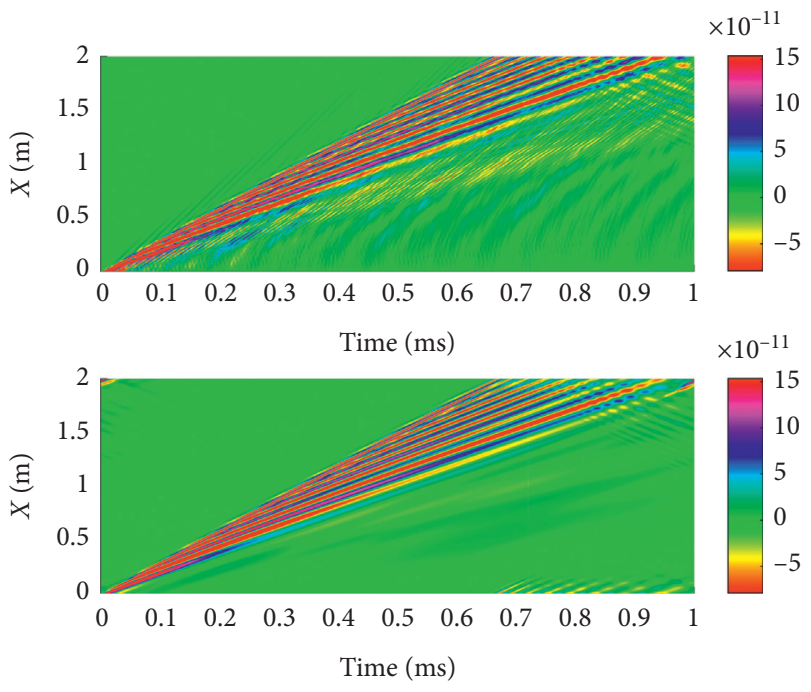

(b)

FIGURE 10: Extracted wavefield of W0 using the selected frequency band. (a) Filtered spectrum and (b) reconstructed time-space domain compared with original wavefield. 
extracted wavefields. The time-space wavefield presented reveals that the W0 mode is almost nondispersive and able to propagate with a long distance along the rail web. The energy is much more focused in waveform after the filtered W0 mode is reconstructed. There is only wave broadening due to the slightly dispersive nature in the relatively large frequency range $25-120 \mathrm{kHz}$, but no more extra wave packets in the signal, denoting that single mode has been extracted. Therefore, the filtering technique and frequency band sweep approach can be applied to extract effective UGW mode under a general broadband excitation along rail web.

\section{Conclusions}

The natures of multimode and dispersion have always been difficult issues in the assessment of integrity of rails based on UGW technology. Due to the complex cross section of rails and continuous train-rail interaction of the railway system, it is not easy to obtain a single mode even with delicate sensor deployment. Moreover, although measured signals with broadband excitation can provide rich information regarding UGW propagation in rails, useful information cannot be extracted in time or frequency domains. In this article, we present a methodology to extract efficient UGW in rails using wavenumber-frequency analysis based on FB theory.

Firstly, dispersive analysis was implemented using WFE based on Floquet-Bloch theory. The complex cross section of rail, as well as the available element types and material library, can be handled with commercially available FE software. By sweeping an incident wavenumber, the dispersive curves were obtained for a rail of 56E1. Then, a 3D FE numerical model was built to investigate the UGW propagation along the rail within the frequency range of $0-120 \mathrm{kHz}$. The time-space wavefield along rail web was analysed by wavenumber-frequency method. The results show the feasibility to verify the dispersion curves and identify specific modes. By carefully designing a 2D bandpass filter based on the predicted dispersion curves and selected wavelength bandwidth $D_{w k}$, it is able to extract and reconstruct dominant mode W0. Further, the frequency range of W0 mode with least dispersion was determined based on a frequency band sweep technique. Thus, a pure and least-dispersive UGW mode was identified and extracted under a broadband excitation. The corresponding optimal frequency range is $25-120 \mathrm{kHz}$. This mode has high potential for evaluation of the integrity of the rail web.

The principle and basic methodology introduced in this paper are also effective for the investigation of other areas of the rail track, such as rail head or rail foot.

\section{Data Availability}

The data used to support the findings of this study are included within the article.

\section{Conflicts of Interest}

The authors declare that there are no conflicts of interest regarding the publication of this paper.

\section{Acknowledgments}

This research was funded by the National Natural Science Foundation of China (51805097), Guangdong Innovative and Entrepreneurial Research Team Program (2016ZT06G375), the Research Grants Council of the Hong Kong Special Administrative Region, China (T32-101/15-R), and National Key R\&D Program of China (2018YFF01010500).

\section{References}

[1] J. Rose, M. Avioli, and W. Song, "Application and potential of guided wave rail inspection," Insight, vol. 44, pp. 353-358, 2002.

[2] J. L. Rose, M. J. Avioli, P. Mudge, and R. Sanderson, "Guided wave inspection potential of defects in rail," NDT \& E International, vol. 37, no. 2, pp. 153-161, 2004.

[3] P. W. Loveday and C. S. Long, "Long range guided wave defect monitoring in rail track," AIP Conference Proceedings, vol. 1581, pp. 179-185, 2014.

[4] G. Yue, X. Cui, K. Zhang, Z. Wang, and D. An, "Guided wave propagation for monitoring the rail base," Mathematical Problems in Engineering, vol. 2020, Article ID 4756574, 11 pages, 2020.

[5] C. Zhou, C. Zhang, and Z. Su, "Health monitoring of rail structures using guided waves and three-dimensional diagnostic imaging," Structural Control \& Health Monitoring, vol. 24, pp. 1-13, 2017.

[6] P. W. Loveday and C. S. Long, "Laser vibrometer measurement of guided wave modes in rail track," Ultrasonics, vol. 57, pp. 209-217, 2015.

[7] T. Hayashi, W.-J. Song, and J. L. Rose, "Guided wave dispersion curves for a bar with an arbitrary cross-section, a rod and rail example," Ultrasonics, vol. 41, no. 3, pp. 175-183, 2003.

[8] W. Yao, F. Sheng, X. Wei, L. Zhang, and Y. Yang, "Propagation characteristics of ultrasonic guided waves in continuously welded rail," Modern Physics Letters B, vol. 31, no. 19-21, Article ID 1740075, 2017.

[9] L. Zhang, X.-R. Gao, W. Wang, and C.-Y. Peng, "The optimization of transducer layout in rail flaw detection," in NDT New Technology \& Application Forum, pp. 120-123, IEEE, Piscataway, NJ, USA, 2015.

[10] D. A. Ramatlo, D. N. Wilke, and P. W. Loveday, "Development of an optimal piezoelectric transducer to excite guided waves in a rail web," NDT \& E International, vol. 95, pp. 72-81, 2018.

[11] P. W. Loveday, "Semi-analytical finite element analysis of elastic waveguides subjected to axial loads," Ultrasonics, vol. 49, no. 3, pp. 298-300, 2009.

[12] I. I. Setshedi, P. W. Loveday, C. S. Long, and D. N. Wilke, "Estimation of rail properties using semi-analytical finite element models and guided wave ultrasound measurements," Ultrasonics, vol. 96, pp. 240-252, 2019.

[13] P. Zuo, X. Yu, and Z. Fan, "Numerical modeling of embedded solid waveguides using SAFE-PML approach using a commercially available finite element package," NDT \& E International, vol. 90, pp. 11-23, 2017.

[14] D. A. Ramatlo, C. S. Long, P. W. Loveday, and D. N. Wilke, “A modelling framework for simulation of ultrasonic guided wave-based inspection of welded rail tracks," Ultrasonics, vol. 108, Article ID 106215, 2020. 
[15] D. J. Thompson, "Experimental analysis of wave propagation in railway tracks," Journal of Sound and Vibration, vol. 203, no. 5, pp. 867-888, 1997.

[16] J. Ryue, D. J. Thompson, P. R. White, and D. R. Thompson, "Investigations of propagating wave types in railway tracks at high frequencies," Journal of Sound and Vibration, vol. 315, no. 1-2, pp. 157-175, 2008.

[17] J. Ryue, D. J. Thompson, P. R. White, and D. Thompson, "Wave propagation in railway tracks at high frequencies," in Noise and Vibration Mitigation for Rail Transportation Systems, pp. 440-446, Springer, Berlin, Germany, 2008.

[18] R. Sanderson and S. Smith, "The application of finite element modelling to guided ultrasonic waves in rails," Insight, vol. 44, pp. 359-363, 2002.

[19] M. Koshiba, S. Mitobe, and M. Suzuki, "Finite-element solution of periodic waveguides for acoustic waves," IEEE Transactions on Ultrasonics, Ferroelectrics and Frequency Control, vol. 34, no. 4, pp. 472-477, 1987.

[20] M. Collet, M. Ouisse, M. Ruzzene, and M. N. Ichchou, "Floquet-Bloch decomposition for the computation of dispersion of two-dimensional periodic, damped mechanical systems," International Journal of Solids and Structures, vol. 48 , no. 20, pp. 2837-2848, 2011.

[21] P. Gómez García and J.-P. Fernández-Álvarez, "Floquet-bloch theory and its application to the dispersion curves of nonperiodic layered systems," Mathematical Problems in Engineering, vol. 2015, Article ID 475364, 12 pages, 2015.

[22] P. D. Wilcox, M. J. S. Lowe, and P. Cawley, "Mode and transducer selection for long range Lamb wave inspection," Journal of Intelligent Materials Systems and Structures, vol. 12, no. 8, pp. 553-565, 2001.

[23] P. W. Loveday and C. S. Long, "Feasibility of detecting cracks in rail track at long range using guided wave ultrasound," in Proceedings of the 9th South African Conference on Computational and Applied Mechanics, Somerset West, South Africa, January 2014.

[24] B. Xing, Z. Yu, X. Xu, L. Zhu, and H. Shi, "Mode selection model for rail crack detection based on ultrasonic guided waves," Shock and Vibration, vol. 2020, Article ID 8045626, 19 pages, 2020.

[25] P. W. Loveday, C. S. Long, and D. A. Ramatlo, "Mode repulsion of ultrasonic guided waves in rails," Ultrasonics, vol. 84, pp. 341-349, 2018.

[26] D. Alleyne and P. Cawley, "A two-dimensional Fourier transform method for the measurement of propagating multimode signals," The Journal of the Acoustical Society of America, vol. 89, no. 3, pp. 1159-1168, 1991.

[27] Z. Tian and L. Yu, "Lamb wave frequency-wavenumber analysis and decomposition," Journal of Intelligent Material Systems and Structures, vol. 25, no. 9, pp. 1107-1123, 2014.

[28] E. V. Andhavarapu, P. Loveday, C. S. Long, and P. S. Heyns, "Accuracy of semi-analytical finite elements for modelling wave propagation in rails," in Proceedings of the Seventh South African Conference on Computational and Applied Mechanics, Pretoria, South Africa, January2010.

[29] S. Kenderian, B. B. Djordjevic, and R. E. Green Jr., "Point and line source laser generation of ultrasound for inspection of internal and surface flaws in rail and structural materials," Research in Nondestructive Evaluation, vol. 13, no. 4, pp. 189-200, 2001.

[30] P. Rajagopal, M. Drozdz, E. A. Skelton, M. J. S. Lowe, and R. V. Craster, "On the use of absorbing layers to simulate the propagation of elastic waves in unbounded isotropic media using commercially available Finite Element packages," NDT \& E International, vol. 51, pp. 30-40, 2012. 